\title{
Nonconstant Periodic Solutions of Discrete $p$-Laplacian System via Clark Duality and Computations of the Critical Groups
}

\author{
Bo Zheng ${ }^{1,2}$ \\ ${ }^{1}$ College of Mathematics and Information Sciences, Guangzhou University, Guangzhou, \\ Guangdong 510006, China \\ ${ }^{2}$ Key Laboratory of Mathematics and Interdisciplinary Sciences of Guangdong Higher Education Institutes, Guangzhou University, \\ Guangzhou, Guangdong 510006, China
}

Correspondence should be addressed to Bo Zheng; zhengbo611@outlook.com

Received 18 October 2013; Accepted 2 January 2014; Published 12 February 2014

Academic Editor: Svatoslav Staněk

Copyright (C) 2014 Bo Zheng. This is an open access article distributed under the Creative Commons Attribution License, which permits unrestricted use, distribution, and reproduction in any medium, provided the original work is properly cited.

We study the existence of periodic solutions to a discrete $p$-Laplacian system. By using the Clark duality method and computing the critical groups, we find a simple condition that is sufficient to ensure the existence of nonconstant periodic solutions to the system.

\section{Introduction}

Let $\mathbb{N}, \mathbb{Z}$, and $\mathbb{R}$ denote the respective sets of natural numbers, integers, and real numbers. For $m, n \in \mathbb{Z}$ with $m \leq n$, write $\mathbb{Z}(m, n)=\mathbb{Z} \cap[m, n]=\{m, m+1, \ldots, n\}$. Let $T \in \mathbb{N}$. We are concerned with the existence of nonconstant $T$-periodic solutions to the following discrete $p$-Laplacian system:

$$
\Delta\left(\phi_{p}(\Delta x(n-1))\right)+\nabla F(n, x(n))=0, \quad n \in \mathbb{Z}(1, T),
$$

where $\Delta$ is the forward difference operator defined by $\Delta x(n)=$ $x(n+1)-x(n)$ and $\phi_{p}$ is the $p$-Laplace operator defined by $\phi_{p}(x)=|x|^{p-2} x(p>1)$. Consider $F \in C^{2}\left(\mathbb{Z} \times \mathbb{R}^{N}, \mathbb{R}\right)$, and $\nabla F(n, x)$ denotes the gradient of $F$ with respect to $x \in \mathbb{R}^{N}$. We assume that $F$ is $T$-periodic in the first variable $n$; that is, $F(n+T, \cdot)=F(n, \cdot)$.

When $p=2$, (1) reduces to the second order discrete $T$ periodic Hamiltonian system:

$$
\Delta^{2} x(n-1)+\nabla F(n, x(n))=0, \quad n \in \mathbb{Z} .
$$

In 2003, Guo and Yu [1] introduced the critical point theory (see, e.g., [2]) to the study of the existence of $T$-periodic solutions of (2). By using Rabinowitz's saddle point theorem, they proved existence of $k T$-periodic solutions, $k \in \mathbb{N}$, when either $\nabla F$ is bounded and $F$ is coercive with respect to $x$ or $F$ satisfies a subquadratic Ambrosetti-Rabinowitz condition and a related coercivity condition. In the same year, they also proved existence of at least two nontrivial $T$-periodic solutions of (2) when $\nabla F$ satisfies a superlinear condition near $x=0$ and $F$ satisfies a superquadratic AmbrosettiRabinowitz condition [3]. The growth condition of $\nabla F$ was later removed in Zhou et al. [4] by using the linking theorem. For additional studies on the existence and multiplicity of solutions to (2) subject to various boundary value conditions through the use of critical point theory, we refer the reader to [4-13].

There have been tremendous efforts devoted to the study of the $p$-Laplacian system (1) and the systems involving the $p$-Laplace operator in recent years [14-20]. Many interesting results have been proved on the existence and multiplicity of solutions to (1) subject to the Dirichlet boundary condition $x(0)=x(T+1)=0$ by using the critical point theory $[16,17,19,21]$. However, we have seen a very limited success in the application of this theory to the study of the existence of periodic solutions for (1). For the general case $p>1, \mathrm{He}$ and Chen [22] obtained a result on the existence of periodic 
solutions for (1) with a convex $F$ by using Clark duality and the perturbation technique. For the case $p \geq 2$, Luo and Zhang [23] proved the existence of nonconstant periodic solutions for

$$
\begin{aligned}
& \Delta\left(\phi_{p}(\Delta x(n-1))\right)-a(n)|x(n)|^{p-2} x(n)+\nabla F(n, x(n)) \\
& \quad=0, \quad n \in \mathbb{Z},
\end{aligned}
$$

by making use of the linking theorem.

Our major goal in this paper is to prove the following theorem which gives a simple sufficient condition for the existence of nonconstant $T$-periodic solutions to the $p$ Laplacian system (1).

Theorem 1. Let $1<p<2$. Assume that $F(n, x)$ is strictly convex in $x$ for every $n \in \mathbb{Z}(1, T)$ and there exist $\alpha>0$ and $\delta>0$ such that, for all $x \in \mathbb{R}^{N}$ and $n \in \mathbb{Z}(1, T)$, one has

$$
\delta \frac{|x|^{q}}{q} \leq F(n, x) \leq \alpha \frac{|x|^{q}}{q}
$$

where $p^{-1}+q^{-1}=1$ with

$$
2 \delta^{p-1} \geq p q^{p-1} \alpha^{p / q}
$$

Then (1) has at least one nonconstant T-periodic solution.

Our assumptions differ from those in $[22,23]$ considerably and can be verified easily. For instance, let $n \in \mathbb{Z}(1, T)$, and

$$
F(n, x)=\frac{(2-\sin n)\left(x_{1}^{2}+x_{2}^{2}+\cdots+x_{N}^{2}\right)^{9 / 2}}{9} .
$$

Then $F(n, x)$ is strictly convex in $x$ for every $n \in \mathbb{Z}(1, T)$, and (4) and (5) are satisfied with $p=9 / 8, q=9, \delta=1$, and $\alpha=3$. For this function $F$, Theorem 1 confirms the existence of at least one nonconstant $T$-periodic solution of (1).

In the remainder of this section, we outline our approach based on the Clark duality and computation of the critical groups. Let $H$ be a real Hilbert space and $f \in C^{1}(H, \mathbb{R})$. In Morse theory, the local behavior of $f$ near an isolated critical point $x_{0}$ at the level $c$ is described by the critical groups:

$$
C_{q}\left(f, x_{0}\right):=H_{q}\left(f^{c} \cap U, f^{c} \cap U \backslash\left\{x_{0}\right\}\right),
$$

where $f^{c}=\{x \in H: f(x) \leq c\}, U$ is a neighbourhood of $x_{0}$ containing no other critical points, and $H$ denotes singular homology. The critical groups distinguish between different types of critical points and are extremely useful for obtaining the existence and multiplicity of solutions for variational problems [24].

Nonzero $T$-periodic solutions of (1) are the nontrivial critical points of the variational functional

$$
\varphi(x)=\sum_{n=1}^{T} \frac{1}{p}|\Delta x(n)|^{p}-F(n, x(n))
$$

defined on the finite dimensional space

$$
\Omega_{T}=\left\{x=\{x(n)\} \mid x(n) \in \mathbb{R}^{N}, x(n+T)=x(n), n \in \mathbb{Z}\right\} ;
$$

see $[9,23]$ for details. However, it is difficult to compute the critical groups for the case $p \neq 2$ because there are few results on the nonlinear eigenvalue problem:

$$
\begin{gathered}
\Delta\left(\phi_{p}(\Delta x(n-1))\right)+\lambda \phi_{p}(x(n))=0, \quad n \in \mathbb{Z}(1, T), \\
x(0)=x(T), \quad x(1)=x(T+1) .
\end{gathered}
$$

As a result, there are no known eigenspaces to work with. As usual, if (10) has nonzero solutions, then we say that $\lambda$ is an eigenvalue of the discrete $p$-Laplacian with periodic boundary condition. To overcome this difficulty, we introduce

$$
u_{1}(n)=x(n), \quad u_{2}(n)=\phi_{p}(\Delta x(n-1))
$$

to transform (1) into the following equivalent first order nonautonomous system:

$$
\begin{aligned}
& \Delta u_{1}(n)=\phi_{q}\left(u_{2}(n+1)\right), \\
& \Delta u_{2}(n)=-\nabla F\left(n, u_{1}(n)\right) .
\end{aligned}
$$

Denote

$$
\begin{gathered}
u(n)=\left(\begin{array}{l}
u_{1}(n) \\
u_{2}(n)
\end{array}\right), \quad J=\left(\begin{array}{cc}
0 & I_{N} \\
-I_{N} & 0
\end{array}\right), \\
L u(n)=\left(\begin{array}{c}
u_{1}(n) \\
u_{2}(n+1)
\end{array}\right) .
\end{gathered}
$$

System (12) can be rewritten in the compact form

$$
J \Delta u(n)+\nabla H(n, L u(n))=0,
$$

where $H(n, u)=H_{1}\left(n, u_{1}\right)+H_{2}\left(n, u_{2}\right)$ with

$$
H_{1}\left(n, u_{1}\right)=F\left(n, u_{1}\right), \quad H_{2}\left(n, u_{2}\right)=\frac{\left|u_{2}\right|^{q}}{q} \text {. }
$$

And nonzero solutions of (14) correspond to nontrivial critical points of

$$
I(u)=\frac{1}{2} \sum_{n=1}^{T}(J \Delta L u(n-1), u(n))+\sum_{n=1}^{T} H(n, L u(n))
$$

defined on

$$
\begin{aligned}
E=\left\{u=\left\{u(n)=\left(\begin{array}{l}
u_{1}(n) \\
u_{2}(n)
\end{array}\right) \in \mathbb{R}^{2 N}\right\} \mid\right. \\
\left.u(n+T)=u(n), u_{i}(n) \in \mathbb{R}^{N}, i=1,2, n \in \mathbb{Z}\right\} .
\end{aligned}
$$

For $u, v \in E, E$ can be equipped with the inner product

$$
\langle u, v\rangle=\sum_{n=1}^{T}(u(n), v(n)),
$$


by which the norm $\|\cdot\|$ can be induced by

$$
\|u\|:=\|u\|_{2}=\left(\sum_{n=1}^{T}|u(n)|^{2}\right)^{1 / 2}
$$

where $|\cdot|$ denotes the Euclidean norm in $\mathbb{R}^{2 N}$ and $(\cdot, \cdot)$ denotes the usual inner product in $\mathbb{R}^{2 N}$. It is easy to know that $E$ is a finite dimensional Hilbert space which can be identified with $\mathbb{R}^{2 T N}$. The variational functional $I$ can be rewritten as

$$
I(u)=\frac{1}{2}\left\langle A_{1} u, u\right\rangle+Q_{1}(u),
$$

where

$$
\begin{gathered}
\left\langle A_{1} u, u\right\rangle=\sum_{n=1}^{T}(J \Delta L u(n-1), u(n)), \\
Q_{1}(u)=\sum_{n=1}^{T} H(n, L u(n)) ;
\end{gathered}
$$

see [11] for details. Very fortunately, the linear eigenvalue problem

$$
\begin{gathered}
J \Delta u(n)+\lambda L u(n)=0, \quad n \in \mathbb{Z}(1, T), \\
u(0)=u(T), \quad u(1)=u(T+1)
\end{gathered}
$$

can be worked out with eigenvalues

$$
\lambda=\lambda_{k}=2 \sin \frac{k \pi}{T}, \quad k \in \mathbb{Z}(-T+1, T-1) .
$$

So, 0 lies in the spectrum of $A_{1}$ which brings another difficulty in computing the critical groups of $I$ at infinity (e.g., to compute the critical groups of $I$ at infinity, the variational functional $I$ may be required to satisfy the angle condition proposed in [25]). To conquer this difficulty, motivated by $[2,9,22]$, we introduce a dual action functional $J$ in the form

$$
J(v)=\frac{1}{2}\langle A v, v\rangle+Q(v)
$$

and 0 is not in the spectrum of $A$. Furthermore, nontrivial critical points of $J$ correspond to nonconstant $T$-periodic solutions of (1). To show that the dual action functional $J$ has at least one nontrivial critical point, firstly, we show that $J$ satisfies the condition $(C)$ which guarantees that the critical groups $C_{*}(J, \infty)$ make sense. Then we compute the critical groups $C_{*}(J, \infty)$. And finally, we show that 0 is a local minimum of $J$ and hence the critical groups at infinity of $J$ are different from the critical groups at zero of $J$ which is sufficient for the existence of at least one nontrivial critical point of $J$ and hence the existence of at least one nonconstant $T$-periodic solution of (1).

\section{The Dual Action Functional and Related Lemmas}

In this section, we present several technical lemmas to facilitate our proof of Theorem 1 in Section 3. In order to decompose the space $E$ appropriately, we consider the eigenvalue problem

$$
\begin{gathered}
J \Delta u(n)+\lambda L u(n)=0, \quad n \in \mathbb{Z}(1, T), \\
u(0)=u(T), \quad u(1)=u(T+1)
\end{gathered}
$$

with $\lambda \in \mathbb{R}$. Apparently, $\lambda=0$ is an eigenvalue of (25) with the eigenfunction

$$
\begin{array}{r}
\eta_{0}(n)=\left(a_{1}, a_{2}, \ldots, a_{2 N}\right)^{\tau}, \quad a_{i} \in \mathbb{R}, \quad i=1,2, \ldots, 2 N, \\
n=1,2, \ldots, T .
\end{array}
$$

Through a simple calculation, we see that (25) is equivalent to

$$
\begin{array}{cc}
\Delta u_{1}(n)=\lambda u_{2}(n+1), & u_{1}(n+T)=u_{1}(n), \\
\Delta u_{2}(n)=-\lambda u_{1}(n), & u_{2}(n+T)=u_{2}(n) .
\end{array}
$$

If $\lambda \neq 0$, then (27) is equivalent to

$$
\begin{array}{ll}
\Delta^{2} u_{1}(n-1)+\lambda^{2} u_{1}(n)=0, & u_{1}(n+T)=u_{1}(n), \\
\Delta^{2} u_{2}(n-1)+\lambda^{2} u_{2}(n)=0, & u_{2}(n+T)=u_{2}(n) .
\end{array}
$$

It has been proved that (28) has a nontrivial solution if and only if $\lambda^{2}=\lambda_{k}^{2}=4 \sin ^{2}(k \pi / T)$ with $k \in \mathbb{Z}(1, T-1)[1,3]$. So in this case (25) has a nontrivial solution if and only if $\lambda=\lambda_{k}=$ $2 \sin (k \pi / T)$ with $k \in \mathbb{Z}(-T+1, T-1) \backslash\{0\}$. The multiplicity of $\lambda_{0}=0$ is $2 N$ and the multiplicities of $\lambda_{k} \neq 0$ are of the same number $N$. So, on the eigenvalue problem (25), the following results hold.

Proposition 2. For the eigenvalue problem (25), the eigenvalues are

$$
\lambda=\lambda_{k}=2 \sin \frac{k \pi}{T}, \quad k \in \mathbb{Z}(-T+1, T-1)
$$

which can be arranged as

$$
\lambda_{-r}<\lambda_{-r+1}<\cdots<\lambda_{-1}<0=\lambda_{0}<\lambda_{1}<\lambda_{2}<\cdots<\lambda_{r}
$$

with $r=(T-1) / 2$ if $T$ is odd, and $r=T / 2$ if $T$ is even.

To make an explicit decomposition of the Hilbert space $E$, we also need to compute the eigenfunctions of (25) corresponding to each $\lambda_{k}, k \neq 0$.

For each fixed $k \in \mathbb{Z}(-r,-1) \cup \mathbb{Z}(1, r)$, a solution of (28) can be written as

$$
\begin{aligned}
& u_{1}(n)=c_{1} \cos (k w n)+c_{2} \sin (k w n), \\
& u_{2}(n)=d_{1} \cos (k w n)+d_{2} \sin (k w n),
\end{aligned}
$$

where $w=2 \pi / T$ and $c_{1}, c_{2}, d_{1}, d_{2}$ are constant vectors in $\mathbb{R}^{N}$. By using the relation between $u_{1}$ and $u_{2}$, that is, (27) with $\lambda=$ $\lambda_{k}$, we obtain

$$
\begin{aligned}
& c_{1} \sin \left(\frac{k w}{2}\right)+c_{2} \cos \left(\frac{k w}{2}\right)=d_{1}, \\
& -c_{1} \cos \left(\frac{k w}{2}\right)+c_{2} \sin \left(\frac{k w}{2}\right)=d_{2} .
\end{aligned}
$$


Let $e_{j}, j=1,2, \ldots, N$, denote the canonical basis of $\mathbb{R}^{N}$. If we choose $c_{1}=e_{j}$ and $c_{2}=0$, then $d_{1}=\sin (k w / 2) e_{j}$, and $d_{2}=-\cos (k w / 2) e_{j}$; if we choose $c_{1}=0$ and $c_{2}=e_{j}$, then $d_{1}=\cos (k w / 2) e_{j}$, and $d_{2}=\sin (k w / 2) e_{j}$. Therefore, the eigenfunctions of (25) corresponding to each $\lambda_{k}(k \neq 0)$ can be given as

$$
\begin{aligned}
& \eta_{k, j}^{(1)}(n)=\left(\begin{array}{c}
\cos (k w n) e_{j} \\
-\sin \left(k w\left(\frac{n-1}{2}\right)\right) e_{j}
\end{array}\right), \quad n=1,2, \ldots, T, \\
& \eta_{k, j}^{(2)}(n)=\left(\begin{array}{c}
\sin (k w n) e_{j} \\
\cos \left(k w\left(\frac{n-1}{2}\right)\right) e_{j}
\end{array}\right), \quad n=1,2, \ldots, T .
\end{aligned}
$$

Let

$$
\begin{aligned}
& W^{0}=\left\{u=\{u(n)\} \mid u(n)=c_{1} e_{1}+c_{2} e_{2}+\cdots\right. \\
& \left.+c_{2 N} e_{2 N}, c_{i} \in \mathbb{R}, i=1,2, \ldots, 2 N\right\} ; \\
& =\left\{u=\{u(n)\} \mid u(n)=\sum_{j=1}^{N} \sum_{k=1}^{r}\left[\alpha_{k, j}^{(1)} \eta_{k, j}^{(1)}(n)+\alpha_{k, j}^{(2)} \eta_{k, j}^{(2)}(n)\right],\right. \\
& \left.\alpha_{k, j}^{(1)}, \alpha_{k, j}^{(2)} \in \mathbb{R}\right\} \\
& =\left\{u=\{u(n)\} \mid u(n)=\sum_{j=1}^{N} \sum_{k=-r}^{-1}\left[\beta_{k, j}^{(1)} \eta_{k, j}^{(1)}(n)+\beta_{k, j}^{(2)} \eta_{k, j}^{(2)}(n)\right],\right. \\
& \left.\beta_{k, j}^{(1)}, \beta_{k, j}^{(2)} \in \mathbb{R}\right\}
\end{aligned}
$$

Note that if $T$ is even and $k=T / 2$, then

$$
\eta_{k, j}^{(1)}(n)=\eta_{k, j}^{(1)}(T-n), \quad \eta_{k, j}^{(2)}(n)=\eta_{k, j}^{(2)}(T-n)
$$

which shorten the dimension of the eigensubspace corresponding to $\lambda_{T / 2}$ to $N$. Hence

$$
\operatorname{dim} W^{0}=2 N, \quad \operatorname{dim} W^{+}=\operatorname{dim} W^{-}=(T-1) N,
$$

and $E$ has a eigensubspace decomposition as

$$
E=W^{0} \oplus W^{-} \oplus W^{+} .
$$

Thus, for any $u \in E, u$ can be expressed in the form

$$
u=\tilde{u}+\bar{u}
$$

where $\tilde{u}=\left(\widetilde{u}_{1}, \widetilde{u}_{2}\right)^{\tau} \in W^{-} \oplus W^{+}$and $\bar{u}=\left(\bar{u}_{1}, \bar{u}_{2}\right)^{\tau} \in W^{0}$. Obviously, $u_{i}=\tilde{u}_{i}+\bar{u}_{i}, i=1,2$.
Denote

$$
\begin{gathered}
\mu_{\min }=\min \left\{\lambda_{k}^{2}=4 \sin ^{2}\left(\frac{k \pi}{T}\right), k \in \mathbb{Z}(1, T-1)\right\}=4 \sin ^{2} \frac{\pi}{T} . \\
\mu_{\max }=\max \left\{\lambda_{k}^{2}=4 \sin ^{2}\left(\frac{k \pi}{T}\right), k \in \mathbb{Z}(1, T-1)\right\} \\
= \begin{cases}4, & T \text { is even; } \\
4 \sin ^{2} \frac{(T-1) \pi}{2 T}, & T \text { is odd. }\end{cases}
\end{gathered}
$$

Then we have the following Wirtinger type inequalities:

$$
\mu_{\min }\left\|\tilde{u}_{i}\right\|^{2} \leq\left\|\Delta \tilde{u}_{i}\right\|^{2} \leq \mu_{\max }\left\|\tilde{u}_{i}\right\|^{2}, \quad i=1,2 .
$$

On the other hand, we can also define the norm $\|\cdot\|_{p}$ on $\mathbb{R}^{2 N T}$ as follows:

$$
\|u\|_{p}=\left(\sum_{n=1}^{T}|u(n)|^{p}\right)^{1 / p}
$$

with $p>1$. Since $\|u\|_{p}$ and $\|u\|$ are equivalent, there exist constants $c_{1}, c_{2}$ such that $c_{2} \geq c_{1}>0$ and

$$
c_{1}\|u\| \leq\|u\|_{p} \leq c_{2}\|u\| .
$$

Now, we introduce the Clark duality $[2,26]$. The Legendre transform $L^{*}(n, \cdot)$ of $L(n, \cdot)$ with respect to the second variable can be defined by

$$
L^{*}(n, y)=\sup _{x \in \mathbb{R}^{M}}\{(x, y)-L(n, x)\},
$$

where $(\cdot, \cdot)$ denotes the usual inner product in $\mathbb{R}^{M}$ with $M$ a given positive integer.

From [2, Theorem 2.2 and Proposition 2.4], we have the following lemma.

Lemma 3. Let, for every $n \in \mathbb{Z}(1, T), L(n, x)$ be continuously differentiable and strictly convex in $x \in \mathbb{R}^{M}$, and

$$
\frac{L(n, x)}{|x|} \longrightarrow+\infty \text { as }|x| \longrightarrow \infty
$$

Then for every $n \in \mathbb{Z}(1, T), L^{*}(n, y)$ is continuously differentiable in $y \in \mathbb{R}^{M}$ and

$$
\begin{aligned}
L^{*}(n, y) & =(x, y)-L(n, x) \\
& \Longleftrightarrow y=\nabla L(n, x) \\
& \Longleftrightarrow x=\nabla L^{*}(n, y) .
\end{aligned}
$$

Remark 4. If, for $x=\left(x_{1}, x_{2}\right)^{\tau}, x_{1}, x_{2} \in \mathbb{R}^{M}, L(n, x)$ can be split into two parts $L(n, x)=L_{1}\left(n, x_{1}\right)+L_{2}\left(n, x_{2}\right)$, then by (45), we have $L^{*}(n, y)=L_{1}^{*}\left(n, y_{1}\right)+L_{2}^{*}\left(n, y_{2}\right), y=\left(y_{1}, y_{2}\right)^{\tau}$, and $y_{1}, y_{2} \in \mathbb{R}^{M}$.

From [2, Proposition 2.2], we have the following lemma. 
Lemma 5. Let convex lower semicontinuous function $L(n, x)$ be such that, for some $\xi>0, t>1, \beta \geq 0$, and $\gamma \geq 0$, one has

$$
-\beta \leq L(n, x) \leq \xi t^{-1}|x|^{t}+\gamma
$$

whenever $x \in \mathbb{R}^{M}$. Then, if

$$
\begin{aligned}
y \in \partial L(n, x)=\left\{\begin{array}{l}
w \\
\in \mathbb{R}^{M}: L(n, z) \geq L(n, x)
\end{array}\right. \\
\left.+(w, z-x) \text { for all } z \in \mathbb{R}^{M}\right\},
\end{aligned}
$$

one has

$$
|y| \leq\left\{s \xi^{s / t}[|x|+\beta+\gamma]+1\right\}^{t-1}
$$

where $s^{-1}+t^{-1}=1$.

Remark 6. From assumption (4) and $p>1$,

$$
\frac{F\left(n, u_{1}\right)}{\left|u_{1}\right|} \longrightarrow+\infty \text { as }\left|u_{1}\right| \longrightarrow \infty
$$

And by Lemma $3, F^{*}\left(n, v_{1}\right)$ is continuously differentiable in $v_{1}$ for every $n \in \mathbb{Z}(1, T)$.

Furthermore, on the $F^{*}\left(n, v_{1}\right)$ we have the following.

Lemma 7. For all $v_{1} \in \mathbb{R}^{N}$ and every $n \in \mathbb{Z}(1, T)$, one has

$$
\begin{gathered}
\alpha^{-p / q} \frac{\left|v_{1}\right|^{p}}{p} \leq F^{*}\left(n, v_{1}\right) \leq \delta^{-p / q} \frac{\left|v_{1}\right|^{p}}{p}, \\
\left|\nabla F^{*}\left(n, v_{1}\right)\right| \leq\left(\frac{q}{\delta}\left|v_{1}\right|+1\right)^{p-1} .
\end{gathered}
$$

Proof. By Lemma 3, $F^{*}\left(n, v_{1}\right)=\left(u_{1}, v_{1}\right)-F\left(n, u_{1}\right) \Leftrightarrow v_{1}=$ $\nabla F\left(n, u_{1}\right)$. Let $F_{1}: \mathbb{R}^{N} \rightarrow \mathbb{R}$ be defined by

$$
F_{1}\left(u_{1}\right)=c \frac{\left|u_{1}\right|^{q}}{q}
$$

where $c>0$. Then

$$
F_{1}^{*}\left(v_{1}\right)=\sup _{u_{1} \in \mathbb{R}^{N}}\left\{\left(v_{1}, u_{1}\right)-c \frac{\left|u_{1}\right|^{q}}{q}\right\}=c^{-p / q} \frac{\left|v_{1}\right|^{p}}{p} .
$$

Hence (4), (53), and the fact that $F_{1}^{*} \geq F_{2}^{*}$ if $F_{1} \leq F_{2}$ give (50).

To show that (51) holds, we can apply Lemma 5 to $F^{*}\left(n, v_{1}\right)$ with $\beta=\gamma=0, \xi=\delta^{-p / q}, p=t$ and $x=v_{1}$, and $y=\nabla F^{*}\left(n, v_{1}\right)$. Then (48) implies that (51) holds.

By Lemmas 3 and 7, $H_{1}^{*}$ and $H_{2}^{*}$ are well defined and

$$
H_{1}^{*}\left(n, v_{1}\right)=F^{*}\left(n, v_{1}\right), \quad H_{2}^{*}\left(n, v_{1}\right)=\frac{\left|v_{1}\right|^{p}}{p} .
$$

The variational functional of (14) defined on $E$ is

$$
I(u)=\frac{1}{2} \sum_{n=1}^{T}(J \Delta L u(n-1), u(n))+\sum_{n=1}^{T} H(n, L u(n))
$$

see [11] for details. If

$$
u(n)=J v(n) \quad \text { or } \quad v(n)=-J u(n),
$$

then, by (45) and (54), we obtain

$$
\begin{aligned}
I(u)= & \frac{1}{2} \sum_{n=1}^{T}(J \Delta u(n-1), u(n))+\sum_{n=1}^{T} H(n, L u(n)) \\
= & \frac{1}{2} \sum_{n=1}^{T}\left[\left(\Delta u_{2}(n), u_{1}(n)\right)-\left(\Delta u_{1}(n-1), u_{2}(n)\right)\right] \\
& +\sum_{n=1}^{T}\left[H_{1}\left(n, u_{1}(n)\right)+H_{2}\left(u_{2}(n+1)\right)\right] \\
= & \sum_{n=1}^{T}\left[\frac{1}{2}\left(\Delta u_{2}(n), u_{1}(n)\right)-\frac{1}{2}\left(\Delta u_{1}(n-1), u_{2}(n)\right)\right. \\
& +\sum_{n=1}^{T}\left[-\left(u_{1}(n), \Delta v_{1}(n)\right)+H_{1}\left(n, u_{1}(n)\right)\right. \\
& -\sum_{n=1}^{T}\left[F^{*}\left(n, \Delta v_{1}(n)\right)+\frac{\left|\Delta v_{2}(n)\right|^{p}}{p}\right], \\
& -\sum_{n=1}^{T}\left[F^{*}\left(n, \Delta v_{1}(n)\right)+\frac{\left|\Delta v_{2}(n)\right|^{p}}{p}\right] \\
= & \frac{1}{2} \sum_{n=1}^{T}\left[\left(\Delta v_{1}(n), v_{2}(n)\right)-\left(\Delta v_{2}(n-1), v_{1}(n)\right)\right] \\
& \\
& \\
&
\end{aligned}
$$

where

$$
\begin{aligned}
F^{*} & \left(n, \Delta v_{1}(n)\right) \\
& =\left(u_{1}(n), \Delta v_{1}(n)\right)-F\left(n, u_{1}(n)\right) \\
& \Longleftrightarrow \Delta v_{1}(n)=\nabla F\left(n, u_{1}(n)\right) \\
& \Longleftrightarrow u_{1}(n)=\nabla F^{*}\left(n, \Delta v_{1}(n)\right) .
\end{aligned}
$$

Now, we introduce a dual action functional

$$
\begin{aligned}
J(v)=\sum_{n=1}^{T} & \frac{1}{2}(L J \Delta v(n-1), v(n)) \\
& +F^{*}\left(n, \Delta v_{1}(n)\right)+\frac{\left|\Delta v_{2}(n)\right|^{p}}{p},
\end{aligned}
$$

where $v=\left(v_{1}, v_{2}\right)^{\tau} \in E$. Note that $J(v)=J(\widetilde{v}+\bar{v})=J(\widetilde{v})$ for $v=\widetilde{v}+\bar{v} \in E$ with $\widetilde{v} \in Y:=W^{+} \oplus W^{-}$and $\bar{v} \in W^{0}$; hence it is 
sufficient to consider the functional $J$ on the subspace $Y$ of $E$. By Remark 6, the functional $J$ is continuously differentiable on $Y$. And, for any $h=\left(h_{1}, h_{2}\right) \in Y$, we have

$$
\begin{aligned}
\left\langle J^{\prime}(v), h\right\rangle & \\
= & \lim _{s \rightarrow 0} \frac{J(v+s h)-J(v)}{s} \\
= & \frac{1}{2} \sum_{n=1}^{T}[(L J \Delta h(n-1), v(n))+(L J \Delta v(n-1), h(n))] \\
& +\sum_{n=1}^{T}\left(\nabla F^{*}\left(n, \Delta v_{1}(n)\right), \Delta h_{1}(n)\right) \\
& +\sum_{n=1}^{T}\left(\phi_{p}\left(\Delta v_{2}(n)\right), \Delta h_{2}(n)\right) \\
= & \sum_{n=1}^{T}\left(-v_{2}(n)+\nabla F^{*}\left(n, \Delta v_{1}(n)\right), \Delta h_{1}(n)\right) \\
& +\left(v_{1}(n+1)+\phi_{p}\left(\Delta v_{2}(n)\right), \Delta h_{2}(n)\right) \\
= & \sum_{n=1}^{T}\left(\Delta\left(v_{2}(n-1)-\nabla F^{*}\left(n-1, \Delta v_{1}(n-1)\right)\right), h_{1}(n)\right) \\
& -\left(\Delta\left(v_{1}(n)+\phi_{p}\left(\Delta v_{2}(n-1)\right)\right), h_{2}(n)\right)
\end{aligned}
$$

If $v$ is a critical point of $J$ on the subspace $Y$, that is, $\left\langle J^{\prime}(v), h\right\rangle=$ 0 for any $h \in Y$, then

$$
\begin{aligned}
& v_{2}(n)=\nabla F^{*}\left(n, \Delta v_{1}(n)\right)-c_{1}, \\
& -v_{1}(n+1)=\phi_{p}\left(\Delta v_{2}(n)\right)-c_{2} .
\end{aligned}
$$

That is,

$$
L J v(n)+c=\nabla H^{*}(n, \Delta v(n)),
$$

where $c=\left(c_{1}, c_{2}\right) \in \mathbb{R}^{2 N}$. Setting $u(n)=\left(u_{1}(n), u_{2}(n)\right)^{\tau}=$ $J v(n)+c$, we get $\Delta u(n)=J \Delta v(n)$, and by relation (45) and (62), $\Delta v(n)=\nabla H(n, L u(n))$. Therefore, $J \Delta u(n)+\nabla H(n, L u(n))=0$. And $u(n+T)=u(n)$ due to $v(n+T)=v(T)$. Hence, we have the following.

Remark 8. If $v \in Y$ is a critical point of the dual action functional $J$, then there exists a constant $c \in \mathbb{R}^{2 N}$ such that $u=\left(u_{1}, u_{2}\right)^{\tau}$ with $u_{1}, u_{2} \in \mathbb{R}^{N}$,

$$
u(n)=J v(n)+c
$$

is a solution of (14), and $u_{1}$ is a solution of (1). If $v$ is a nontrivial critical point, then $u$ is a nonconstant $T$-periodic solution of (14), and $u_{1}$ is a nonconstant $T$-periodic solution of (1).

\section{Proof of the Main Result}

As our proof of Theorem 1 is mainly based on the computation of the critical groups in Morse theory, we recall several basic concepts about critical groups $[2,24]$. Let $H$ be a real Hilbert space, and $f \in C^{1}(H, \mathbb{R})$. Denote

$$
\begin{gathered}
f^{c}=\{x \in H: f(x) \leq c\}, \\
\mathscr{K}_{c}=\left\{x \in H: f^{\prime}(x)=0, f(x)=c\right\}
\end{gathered}
$$

for $c \in \mathbb{R}$.

Definition 9. The functional $f$ satisfies the Palais-Smale (PS) condition if any sequence $\left\{x_{m}\right\} \subset H$ such that $\left\{f\left(x_{m}\right)\right\}$ is bounded and $f^{\prime}\left(x_{m}\right) \rightarrow 0$ as $m \rightarrow \infty$ has a convergent subsequence.

In [27], Cerami introduced a weak version of the (PS) condition as follows.

Definition 10. The functional $f$ satisfies the Cerami condition (the $(C)$ condition for short) if any sequence $\left\{x_{m}\right\} \subset H$ such that $\left\{f\left(x_{m}\right)\right\}$ is bounded and $\left(1+\left\|x_{m}\right\|\right)\left\|f^{\prime}\left(x_{m}\right)\right\| \rightarrow 0$ as $m \rightarrow \infty$ has a convergent subsequence.

If $f$ satisfies the (PS) condition or the $(C)$ condition, then $f$ also satisfies the following deformation condition which is essential in Morse theory [28, 29].

Definition 11 (deformation condition). The functional $f$ satisfies the $\left(D_{c}\right)$ condition at the level $c \in \mathbb{R}$ if, for any $\bar{\epsilon}>0$ and any neighborhood $\mathscr{N}$ of $\mathscr{K}_{c}$, there are $\epsilon>0$ and a continuous deformation $\eta:[0,1] \times H \rightarrow H$ such that

(1) $\eta(0, x)=x$ for all $x \in H$;

(2) $\eta(t, x)=x$ for all $x \notin f^{-1}([c-\bar{\epsilon}, c+\bar{\epsilon}])$;

(3) $f(\eta(t, x))$ is nonincreasing in $t$ for any $x \in H$;

(4) $\eta\left(1, f^{c+\epsilon} \backslash \mathcal{N}\right) \subset f^{c-\epsilon}$.

$f$ satisfies the $(D)$ condition if $f$ satisfies the $\left(D_{c}\right)$ condition for all $c \in \mathbb{R}$.

Let $x_{0}$ be an isolated critical point of $f$ with $f\left(x_{0}\right)=c \epsilon$ $\mathbb{R}$, and let $U$ be a neighborhood of $x_{0}$; the group

$$
C_{q}\left(f, x_{0}\right):=H_{q}\left(f^{c} \cap U, f^{c} \cap U \backslash\left\{x_{0}\right\}\right), \quad q \in \mathbb{Z}
$$

is called the $q$ th critical group of $f$ at $x_{0}$, where $H_{q}(A, B)$ denotes the $q$ th singular relative homology group of the pair $(A, B)$ over a field $\mathfrak{F}$, which is defined to be quotient $H_{q}(A, B)=Z_{q}(A, B) / B_{q}(A, B)$, where $Z_{q}(A, B)$ is the $q$ th singular relative closed chain group and $B_{q}(A, B)$ is the $q$ th singular relative boundary chain group [30].

Bartsch and $\mathrm{Li}$ [25] defined the qth critical group of $f$ at infinity as

$$
C_{q}(f, \infty):=H_{q}\left(H, f^{a}\right), \quad q \in \mathbb{Z},
$$

provided that $f(\mathscr{K})$ is bounded from below by $a \in \mathbb{R}$ with $\mathscr{K}=\left\{x \in E: f^{\prime}(x)=0\right\}$ and $f$ satisfies the $\left(D_{c}\right)$ condition for all $c \leq a$.

Assume $\sharp \mathscr{K}<\infty$ and $f$ satisfies the $(D)$ condition. The Morse-type numbers of the pair $\left(H, f^{a}\right)$ are defined by

$$
M_{q}=M_{q}\left(H, f^{a}\right)=\sum_{x \in \mathscr{K}} \operatorname{dim} C_{q}(f, x),
$$


and the Betti numbers of the pair $\left(H, f^{a}\right)$ are

$$
\beta_{q}:=\operatorname{dim} C_{q}(f, \infty)
$$

see $[2,24]$. Furthermore, the following relations hold:

$$
\begin{gathered}
\sum_{j=0}^{q}(-1)^{q-j} M_{j} \geq \sum_{j=0}^{q}(-1)^{q-j} \beta_{j}, \quad q \in \mathbb{Z}, \\
\sum_{q=0}^{\infty} M_{q}=\sum_{q=0}^{\infty} \beta_{q} .
\end{gathered}
$$

Thus, if $C_{q}(f, \infty) \not 0$, that is, $\beta_{q} \neq 0$ for some $q \in \mathbb{Z}$, then there must exist a critical point $x$ of $f$ with $C_{q}(f, x) \not 0$. Furthermore, the following results hold.

Proposition 12 (see [24]). Let $H$ be a real Hilbert space and $f \in C^{2}(H, \mathbb{R})$. Assume that $\sharp \mathscr{K}<\infty$ and that $f$ satisfies the (D) condition. If there exists some $q \in \mathbb{Z}$ such that

(i) $C_{q}(f, \infty) \not 0$, then $f$ must have a critical point $x$ with $C_{q}(f, x) \neq 0$,

(ii) $C_{q}(f, 0) \not C_{q}(f, \infty)$, then $f$ must have a nontrivial critical point.

We will use the following result to compute the critical groups of $J$ at infinity.

Proposition 13 (see [24]). Let the functional $f: H \rightarrow \mathbb{R}$ be of the form

$$
f(x)=\frac{1}{2}\langle A x, x\rangle+Q(x),
$$

where $A: H \rightarrow H$ is a self-adjoint linear operator such that 0 is not in the spectrum of $A, V^{ \pm}$are invariant subspaces corresponding to the positive/negative of spectrum of $A$, respectively, $A^{ \pm}:=\left.A\right|_{V^{ \pm}}$has a bounded inverse on $V^{ \pm}$, and $Q \in C^{1}(H, \mathbb{R})$ has a compact differential $Q^{\prime}(x)$ with

$$
\lim _{\|x\| \rightarrow \infty} \frac{\left\|Q^{\prime}(x)\right\|}{\|x\|}=0 .
$$

Assume that $m=\operatorname{dim} V^{-}$is finite and $f$ satisfies the deformation condition. Then

$$
C_{q}(f, \infty)=\delta_{q, m} \mathfrak{\mho}, \quad q \in \mathbb{Z} .
$$

For the proof of Theorem 1, in what follows we may assume that $J$ has only finitely many critical points. Firstly, we show that $J$ satisfies the condition $(C)$ which guarantees that the critical groups $C_{*}(J, \infty)$ of $J$ at infinity make sense. Then, via computations of critical groups of $J$ at infinity and at zero, we complete the proof of Theorem 1 .

Lemma 14. Under the conditions of Theorem 1, the functional $J$ defined by (59) satisfies (C) condition.

Proof. Let $\left\{v^{(l)}\right\} \subset Y$ be a Cerami sequence of $J$. Since $\operatorname{dim} Y$ is finite, we only need to show that $\left\{v^{(l)}\right\}$ is bounded.
If $\left\{v^{(l)}\right\}$ is unbounded, up to a subsequence, still denoted by $\left\{v^{(l)}\right\}$, we may assume that, for some $c \in \mathbb{R}$,

$$
J\left(v^{(l)}\right) \longrightarrow c, \quad\left\|v^{(l)}\right\| \longrightarrow \infty, \quad\left\|J^{\prime}\left(v^{(l)}\right)\right\|\left\|v^{(l)}\right\| \longrightarrow 0 .
$$

In particular,

$$
\lim _{l \rightarrow \infty}\left(J\left(v^{(l)}\right)-\frac{1}{2}\left\langle J^{\prime}\left(v^{(l)}\right), v^{(l)}\right\rangle\right)=c .
$$

However, by (50) and (51), we have

$$
\begin{aligned}
& J\left(v^{(l)}\right)-\frac{1}{2}\left\langle J^{\prime}\left(v^{(l)}\right), v^{(l)}\right\rangle \\
&=\sum_{n=1}^{T} F^{*}\left(n, \Delta v_{1}^{(l)}(n)\right)-\frac{1}{2}\left(\nabla F^{*}\left(n, \Delta v_{1}^{(l)}(n)\right), \Delta v_{1}^{(l)}(n)\right) \\
&+\left(\frac{1}{p}-\frac{1}{2}\right)\left|\Delta v_{2}^{(l)}(n)\right|^{p} \\
& \geq \sum_{n=1}^{T}\left[\frac{\alpha^{-p / q}}{p}-\frac{1}{2}\left(\frac{q}{\delta}\right)^{p-1}\right]\left|\Delta v_{1}^{(l)}(n)\right|^{p} \\
&+\left(\frac{1}{p}-\frac{1}{2}\right)\left|\Delta v_{2}^{(l)}(n)\right|^{p} .
\end{aligned}
$$

By relation (11) and $u^{(l)}(n)=J v^{(l)}(n)$, one has

$$
v_{1}^{(l)}(n)=-\phi_{p}(\Delta(x(n-1))), \quad v_{2}^{(l)}(n)=x(n),
$$

which implies that $v_{1}^{(l)}(n)=-\phi_{p}\left(\Delta\left(v_{2}^{(l)}(n-1)\right)\right)$, and hence

$$
\left\|v_{2}^{(l)}\right\| \longrightarrow \infty \quad \text { as }\left\|v^{(l)}\right\| \longrightarrow \infty .
$$

At the same time, note that

$$
\sum_{n=1}^{T}\left|\Delta v_{2}^{(l)}(n)\right|^{p}=\left\|\Delta v_{2}^{(l)}\right\|_{p}^{p} \geq c_{1}^{p}\left\|\Delta v_{2}^{(l)}\right\|^{p} \geq c_{1}^{p} \mu_{\min }^{p / 2}\left\|v_{2}^{(l)}\right\|^{p},
$$

so,

$$
\sum_{n=1}^{T} \frac{1}{q}\left|\Delta v_{2}^{(l)}(n)\right|^{p} \longrightarrow \infty \quad \text { as }\left\|v^{(l)}\right\| \longrightarrow \infty
$$

And assumption (5) implies that

$$
\frac{\alpha^{-p / q}}{p}-\frac{1}{2}\left(\frac{q}{\delta}\right)^{p-1} \geq 0
$$

so

$$
\lim _{l \rightarrow \infty}\left(J\left(v^{(l)}\right)-\frac{1}{2}\left\langle J^{\prime}\left(v^{(l)}\right), v^{(l)}\right\rangle\right)=+\infty
$$

holds which gives a contradiction to (74). This completes the proof. 
Lemma 15. Under the conditions of Theorem $1, C_{q}(J, \infty) \cong$ $\delta_{q, l} \mathfrak{F}$ with $l=(T-1) N, q \in \mathbb{Z}$.

Proof. Recall the dual action functional $J: Y \rightarrow \mathbb{R}$ being of the form

$$
\begin{aligned}
J(v)=\sum_{n=1}^{T} & \frac{1}{2}(L J \Delta v(n-1), v(n)) \\
& +F^{*}\left(n, \Delta v_{1}(n)\right)+\frac{\left|\Delta v_{2}(n)\right|^{p}}{p} .
\end{aligned}
$$

For any $\tilde{u}, v \in Y$, if we define a bilinear function as $a(\widetilde{u}, v)=$ $\sum_{n=1}^{T}(L J \Delta \widetilde{u}(n-1), v(n))$, then by $(40)$ one has

$$
\begin{aligned}
|a(\tilde{u}, v)| & \leq\left(\sum_{n=1}^{T}|L J \Delta \widetilde{u}(n-1)|^{2}\right)^{1 / 2}\left(\sum_{n=1}^{T}|v(n)|^{2}\right)^{1 / 2} \\
& =\left(\sum_{n=1}^{T}|\Delta \widetilde{u}(n)|^{2}\right)^{1 / 2}\left(\sum_{n=1}^{T}|v(n)|^{2}\right)^{1 / 2} \\
& \leq \sqrt{\mu_{\max }}\|\tilde{u}\|\|v\| .
\end{aligned}
$$

By Riese representation theorem [31], we can define the unique continuous self-adjoint linear operator $A$ on $Y$ by $\langle A v, v\rangle=\sum_{n=1}^{T}(L J \Delta v(n-1), v(n))$. If $\lambda$ is in the spectrum of $A$, then the equation $L J \Delta v(n-1)=\lambda v(n)$ yields nontrivial solutions in $E$ which turns out to be the same as (28) with $u$ being replaced by $v$ and the same invariant subspaces $W^{+}$ and $W^{-}$which can be given by eigensubspace. It is obvious that 0 is not in the spectrum of $A$ from the definition of the subspaces of $W^{+}$and $W^{-}$. Hence, by Proposition 13 , we only need to prove that

$$
\left\|Q^{\prime}(v)\right\|=o(\|v\|) \quad \text { as }\|v\| \longrightarrow \infty
$$

where

$$
Q(v)=\sum_{n=1}^{T} F^{*}\left(n, \Delta v_{1}(n)\right)+\frac{1}{p}\left|\Delta v_{2}(n)\right|^{p} .
$$

Note that

$$
\begin{aligned}
\left\langle Q^{\prime}(v), h\right\rangle=\sum_{n=1}^{T} & \left(\nabla F^{*}\left(n, \Delta v_{1}(n)\right), \Delta h_{1}(n)\right) \\
& +\left(\left|\Delta v_{2}(n)\right|^{p-2} \Delta v_{2}(n), \Delta h_{2}(n)\right)
\end{aligned}
$$

with $h(n)=\left(h_{1}(n), h_{2}(n)\right)^{\tau} \in Y$. And

$$
\begin{aligned}
& \lim _{\|v\| \rightarrow \infty} \frac{\left\langle Q^{\prime}(v), v\right\rangle}{\|v\|^{2}} \\
& =\lim _{\|v\| \rightarrow \infty} \sum_{n=1}^{T} \frac{\left(\nabla F^{*}\left(n, \Delta v_{1}(n)\right), \Delta v_{1}(n)\right)}{\|v\|^{2}} \\
& +\frac{\left(\left|\Delta v_{2}(n)\right|^{p-2} \Delta v_{2}(n), \Delta v_{2}(n)\right)}{\|v\|^{2}} \\
& =\lim _{\|v\| \rightarrow \infty} \sum_{n=1}^{T} \frac{\left(\nabla F^{*}\left(n, \Delta v_{1}(n)\right), \Delta v_{1}(n)\right)}{\left\|\Delta v_{1}\right\|^{2}} \frac{\left\|\Delta v_{1}\right\|^{2}}{\|v\|^{2}} \\
& +\frac{\left(\left|\Delta v_{2}(n)\right|^{p-2} \Delta v_{2}(n), \Delta v_{2}(n)\right)}{\left\|\Delta v_{2}\right\|^{2}} \frac{\left\|\Delta v_{2}\right\|^{2}}{\|v\|^{2}} .
\end{aligned}
$$

By (51), $1<p<2$, and the equivalence of $\|v\|$ and $\|\Delta v\|$, we see that $(84)$ holds. Hence $C_{q}(J, \infty)=\delta_{q,(T-1) N} \mathfrak{F}$ by Proposition 13 and (36).

Proof of Theorem 1. First, we prove that 0 is a local minimum of $J$ and, hence,

$$
C_{q}(J, 0) \cong \delta_{q, 0} \mathfrak{F}
$$

Firstly, by (40), for all $v \in Y$, we have

$$
\begin{aligned}
& \sum_{n=1}^{T}(L J \Delta v(n-1), v(n)) \\
& \quad \geq-\left(\sum_{n=1}^{T}|L J \Delta v(n-1)|^{2}\right)^{1 / 2}\left(\sum_{n=1}^{T}|v(n)|^{2}\right)^{1 / 2} \\
& \quad=-\left(\sum_{n=1}^{T}|\Delta v(n)|^{2}\right)^{1 / 2}\left(\sum_{n=1}^{T}|v(n)|^{2}\right)^{1 / 2} \\
& \quad \geq-\mu_{\max }^{1 / 2} \sum_{n=1}^{T}|v(n)|^{2} \geq-2\|v\|^{2} .
\end{aligned}
$$

Hence, by (40), (42), and (50), for $v \in Y$, we have

$$
J(v)=\sum_{n=1}^{T} \frac{1}{2}(L J \Delta v(n-1), v(n))
$$

$$
\begin{gathered}
+F^{*}\left(n, \Delta v_{1}(n)\right)+\frac{\left|\Delta v_{2}(n)\right|^{p}}{p} \\
\geq-\sum_{n=1}^{T}|v(n)|^{2}+\alpha^{-p / q} \sum_{n=1}^{T} \frac{\left|\Delta v_{1}(n)\right|^{p}}{p}+\sum_{n=1}^{T} \frac{\left|\Delta v_{2}(n)\right|^{p}}{p} \\
\geq-\|v\|^{2}+\frac{\alpha^{-p / q} c_{1}^{p}}{p}\left\|\Delta v_{1}\right\|^{p}+\frac{c_{1}^{p}}{p}\left\|\Delta v_{2}\right\|^{p}
\end{gathered}
$$




$$
\begin{aligned}
\geq & -\|v\|^{2}+\frac{\alpha^{-p / q} c_{1}^{p} \mu_{\mathrm{min}}^{p / 2}}{p}\left\|v_{1}\right\|^{p}+\frac{c_{1}^{p} \mu_{\mathrm{min}}^{p / 2}}{p}\left\|v_{2}\right\|^{p} \\
= & -\left\|v_{1}\right\|^{2}-\left\|v_{2}\right\|^{2}+\frac{\alpha^{-p / q} c_{1}^{p} \mu_{\mathrm{min}}^{p / 2}}{p}\left\|v_{1}\right\|^{p}+\frac{c_{1}^{p} \mu_{\mathrm{min}}^{p / 2}}{p}\left\|v_{2}\right\|^{p} \\
= & \left\|v_{1}\right\|^{p}\left(\frac{\alpha^{-p / q} c_{1}^{p} \mu_{\mathrm{min}}^{p / 2}}{p}-\left\|v_{1}\right\|^{2-p}\right) \\
& +\left\|v_{2}\right\|^{p}\left(\frac{c_{1}^{p} \mu_{\min }^{p / 2}}{p}-\left\|v_{2}\right\|^{2-p}\right) .
\end{aligned}
$$

Take

$$
\delta=\min \left\{\left(\frac{\alpha^{-p / q} c_{1}^{p} \mu_{\min }^{p / 2}}{2 p}\right)^{1 /(2-p)},\left(\frac{c_{1}^{p} \mu_{\min }^{p / 2}}{2 p}\right)^{1 /(2-p)}, 1\right\} .
$$

Then, for $\|v\| \leq \delta$, one has

$$
\begin{aligned}
J(v) & \geq \delta\left(\left\|v_{1}\right\|^{p}+\left\|v_{2}\right\|^{p}\right) \geq \delta\left(\left\|v_{1}\right\|^{2}+\left\|v_{2}\right\|^{2}\right) \\
& =\delta\|v\|^{2} \geq 0=J(0) .
\end{aligned}
$$

Hence 0 is a local minimum of $J$, and (88) must hold. By Lemma 15, (88), and Proposition 12, J must have at least one nontrivial critical point. The proof is completed.

\section{Conflict of Interests}

The author declares that there is no conflict of interests regarding the publication of this paper.

\section{Acknowledgments}

This project is supported by the National Natural Science Foundation of China (no. 11301103), SRF of Guangzhou Education Bureau (no. 10A012), Program for Changjiang Scholars and Innovative Research Team in University (no. IRT1226), and the Projects for Outstanding Young Teachers and High Level Talents of Guangdong Higher Education Institutes.

\section{References}

[1] Z. Guo and J. Yu, "The existence of periodic and subharmonic solutions of subquadratic second order difference equations," Journal of the London Mathematical Society, vol. 68, no. 2, pp. 419-430, 2003.

[2] J. Mawhin and M. Willem, Critical Point Theory and Hamiltonian Systems, vol. 74 of Applied Mathematical Sciences, Springer, New York, NY, USA, 1989.

[3] Z. Guo and J. Yu, "Existence of periodic and subharmonic solutions for second-order superlinear difference equations," Science in China A, vol. 46, no. 4, pp. 506-515, 2003.

[4] Z. Zhou, J. Yu, and Z. Guo, "Periodic solutions of higher-dimensional discrete systems," Proceedings of the Royal Society of Edinburgh A, vol. 134, no. 5, pp. 1013-1022, 2004.
[5] H.-H. Bin, J.-S. Yu, and Z.-M. Guo, "Nontrivial periodic solutions for asymptotically linear resonant difference problem," Journal of Mathematical Analysis and Applications, vol. 322, no. 1, pp. 477-488, 2006.

[6] X. Deng, H. Shi, and X. Xie, "Periodic solutions of second order discrete Hamiltonian systems with potential indefinite in sign," Applied Mathematics and Computation, vol. 218, no. 1, pp. 148156, 2011.

[7] H. Shi, "Boundary value problems of second order nonlinear functional difference equations," Journal of Difference Equations and Applications, vol. 16, no. 9, pp. 1121-1130, 2010.

[8] Y.-F. Xue and C.-L. Tang, "Existence of a periodic solution for subquadratic second-order discrete Hamiltonian system," Nonlinear Analysis. Theory, Methods \& Applications A, vol. 67, no. 7, pp. 2072-2080, 2007.

[9] J. Yu, H. Bin, and Z. Guo, "Multiple periodic solutions for discrete Hamiltonian systems," Nonlinear Analysis. Theory, Methods \& Applications A, vol. 66, no. 7, pp. 1498-1512, 2007.

[10] J. Yu, Y. Long, and Z. Guo, "Subharmonic solutions with prescribed minimal period of a discrete forced pendulum equation," Journal of Dynamics and Differential Equations, vol. 16, no. 2, pp. 575-586, 2004.

[11] B. Zheng, "Multiple periodic solutions to nonlinear discrete Hamiltonian systems," Advances in Difference Equations, vol. 2007, Article ID 41830, 13 pages, 2007.

[12] Z. Zhou, J. Yu, and Y. Chen, "On the existence of gap solitons in a periodic discrete nonlinear Schrödinger equation with saturable nonlinearity," Nonlinearity, vol. 23, no. 7, pp. 17271740, 2010.

[13] Z. Zhou, J. Yu, and Y. Chen, "Periodic solutions of a $2 n$ th-order nonlinear difference equation," Science China, vol. 53, no. 1, pp. 41-50, 2010.

[14] S. Amghibech, "Bounds for the largest $p$-Laplacian eigenvalue for graphs," Discrete Mathematics, vol. 306, no. 21, pp. 27622771, 2006.

[15] R. P. Agarwal, D. O’Regan, and S. Stanêk, “An existence principle for nonlocal difference boundary value problems with $\varphi$ Laplacian and its application to singular problems," Advances in Difference Equations, vol. 2008, Article ID 154302, 10 pages, 2008.

[16] R. P. Agarwal, K. Perera, and D. O’Regan, "Multiple positive solutions of singular discrete $p$-Laplacian problems via variational methods," Advances in Difference Equations, no. 2, pp. 93 99, 2005.

[17] P. Candito and N. Giovannelli, "Multiple solutions for a discrete boundary value problem involving the $p$-Laplacian," Computers \& Mathematics with Applications, vol. 56, no. 4, pp. 959-964, 2008.

[18] Z. He, "On the existence of positive solutions of $p$-Laplacian difference equations," Journal of Computational and Applied Mathematics, vol. 161, no. 1, pp. 193-201, 2003.

[19] L. Jiang and Z. Zhou, "Three solutions to Dirichlet boundary value problems for $p$-Laplacian difference equations," Advances in Difference Equations, vol. 2008, Article ID 345916, 10 pages, 2008.

[20] J.-H. Park and S.-Y. Chung, "The Dirichlet boundary value problems for $p$-Schrödinger operators on finite networks," Journal of Difference Equations and Applications, vol. 17, no. 5, pp. 795-811, 2011.

[21] G. Bonanno and P. Candito, "Nonlinear difference equations investigated via critical point methods," Nonlinear Analysis. 
Theory, Methods \& Applications A, vol. 70, no. 9, pp. 3180-3186, 2009.

[22] T. He and W. Chen, "Periodic solutions of second order discrete convex systems involving the $p$-Laplacian," Applied Mathematics and Computation, vol. 206, no. 1, pp. 124-132, 2008.

[23] Z. Luo and X. Zhang, "Existence of nonconstant periodic solutions for a nonlinear discrete system involving the $p$-Laplacian," Bulletin of the Malaysian Mathematical Sciences Society, vol. 35, no. 2, pp. 373-382, 2012.

[24] K. Chang, Infinite-Dimensional Morse Theory and Multiple Solution Problems, vol. 6 of Progress in Nonlinear Differential Equations and their Applications, Birkhäuser, Boston, Mass, USA, 1993.

[25] T. Bartsch and S. Li, "Critical point theory for asymptotically quadratic functionals and applications to problems with resonance," Nonlinear Analysis. Theory, Methods \& Applications A, vol. 28, no. 3, pp. 419-441, 1997.

[26] F. H. Clarke and I. Ekeland, "Hamiltonian trajectories having prescribed minimal period," Communications on Pure and Applied Mathematics, vol. 33, no. 2, pp. 103-116, 1980.

[27] G. Cerami, "An existence criterion for the critical points on unbounded manifolds," Istituto Lombardo. Accademia di Scienze e Lettere. Rendiconti. Scienze Matematiche, Fisiche, Chimiche e Geologiche A, vol. 112, no. 2, pp. 332-336, 1978.

[28] P. Bartolo, V. Benci, and D. Fortunato, "Abstract critical point theorems and applications to some nonlinear problems with "strong" resonance at infinity," Nonlinear Analysis. Theory, Methods \& Applications A, vol. 7, no. 9, pp. 981-1012, 1983.

[29] K. C. Chang, "Solutions of asymptotically linear operator equations via Morse theory," Communications on Pure and Applied Mathematics, vol. 34, no. 5, pp. 693-712, 1981.

[30] E. Spanier, Algebraic Topology, Springer, 1994.

[31] M. Reed and B. I. Simon, Functional Analysis, Elsevier, 1981. 


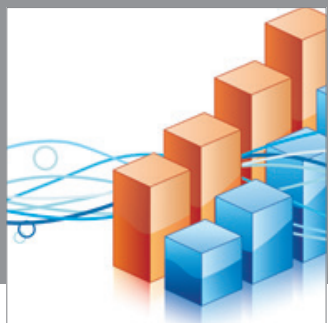

Advances in

Operations Research

mansans

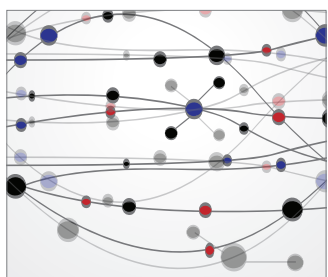

The Scientific World Journal
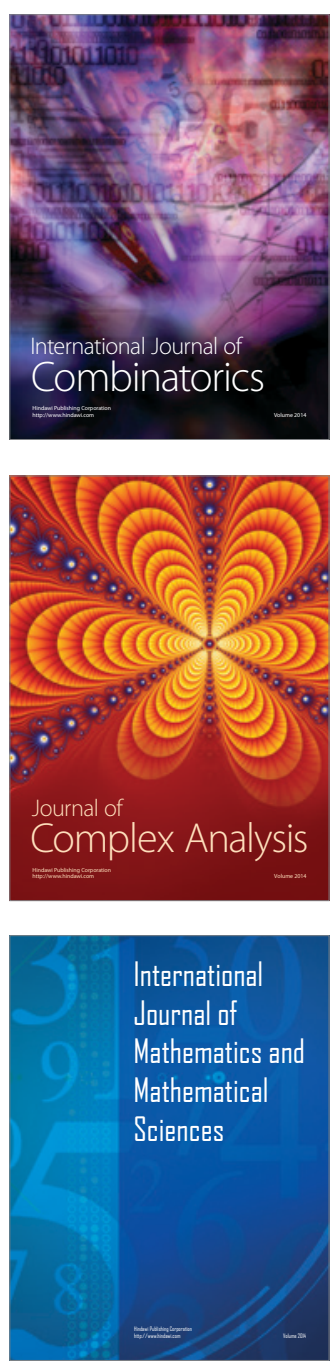
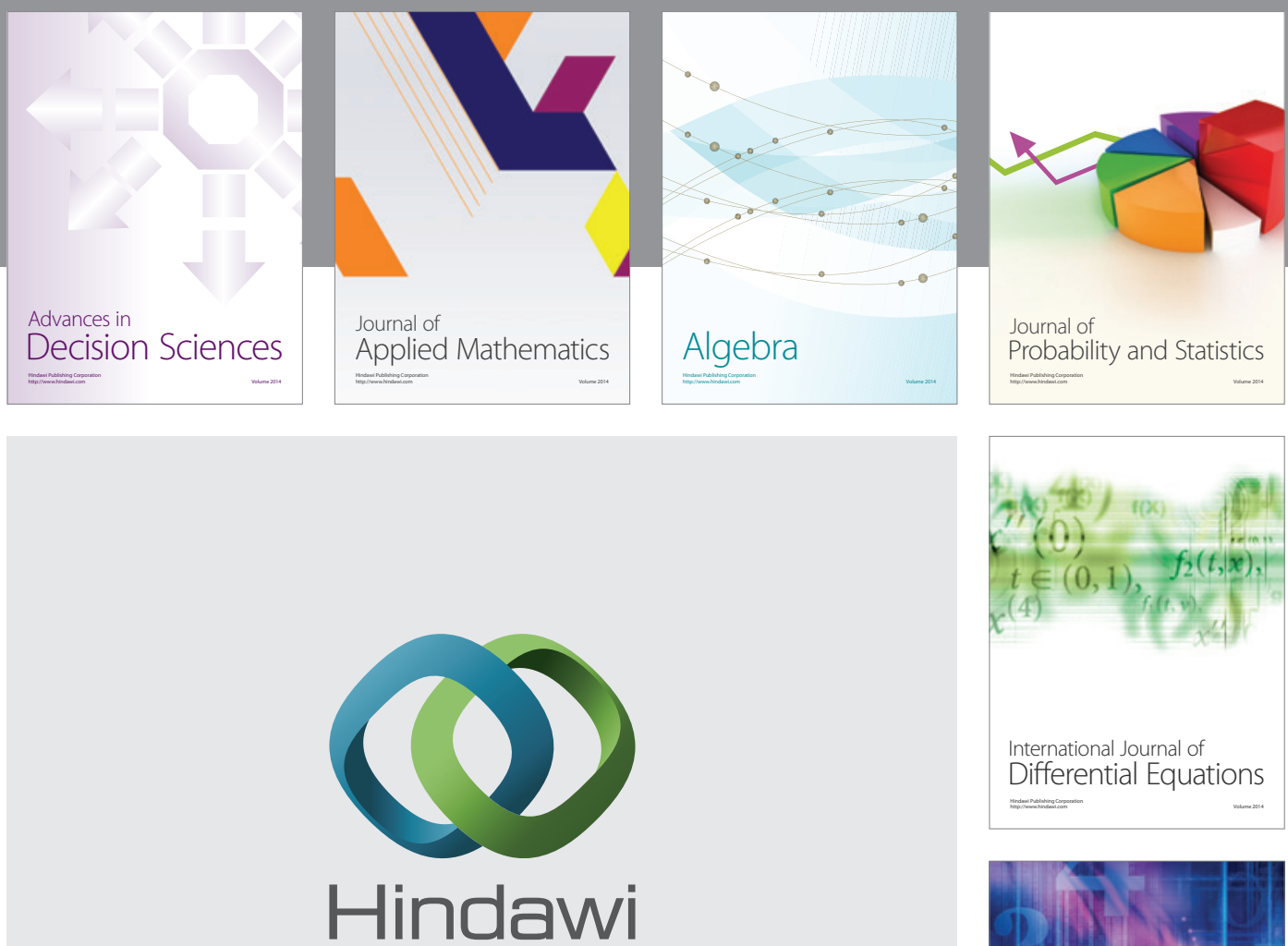

Submit your manuscripts at http://www.hindawi.com
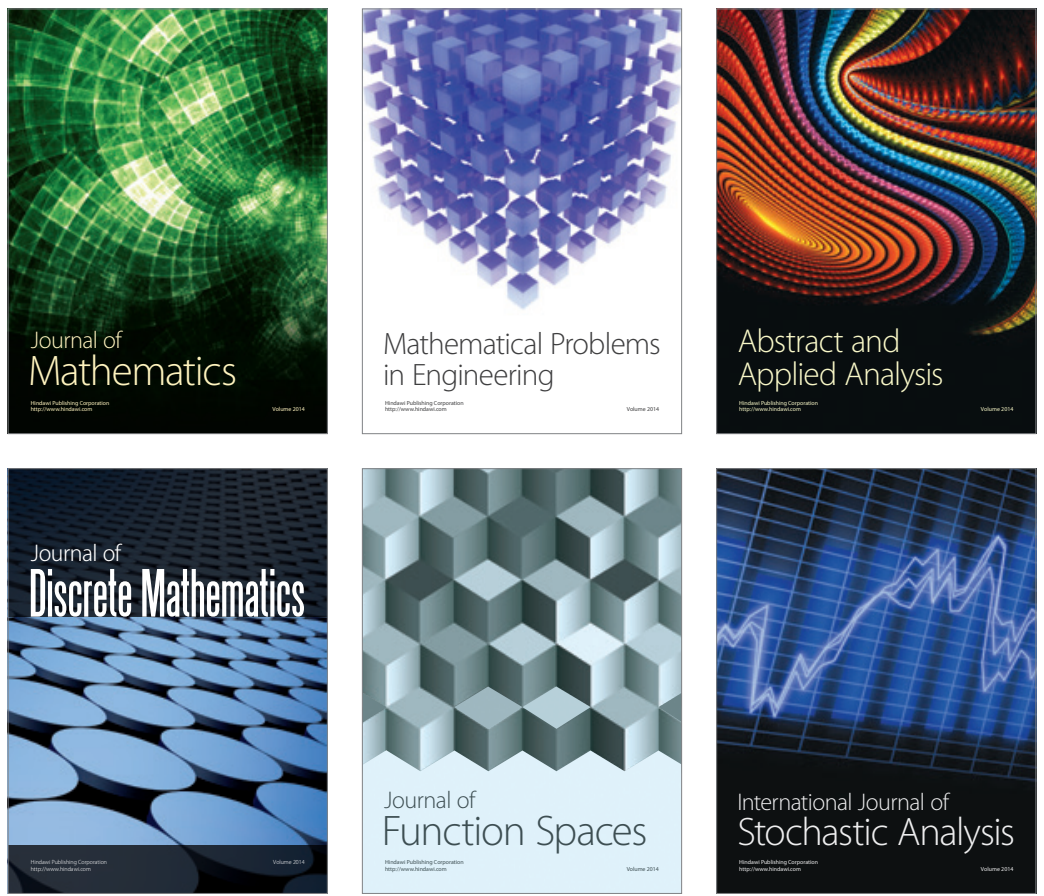

Journal of

Function Spaces

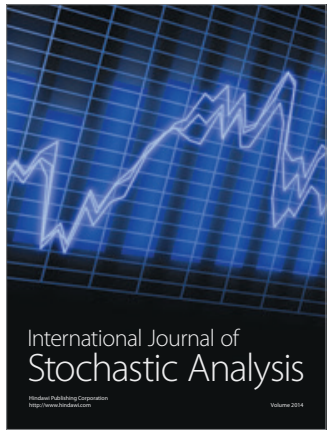

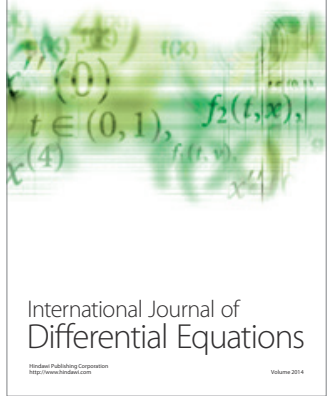
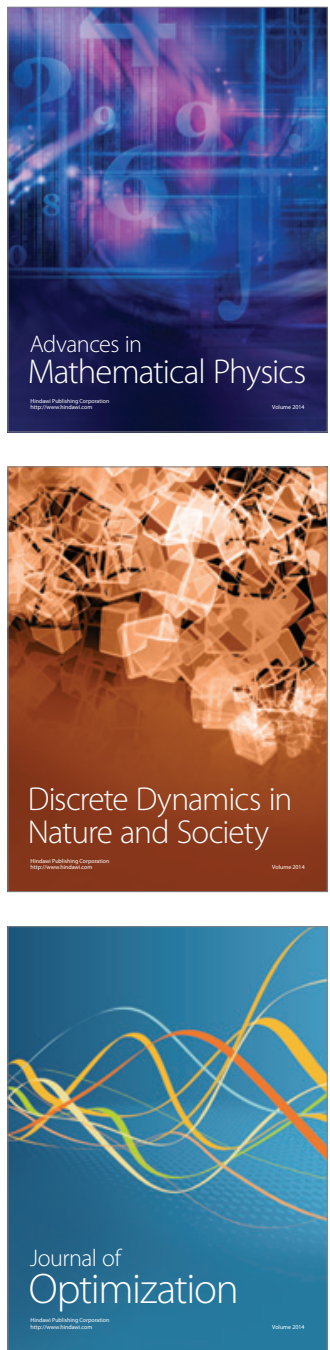